\title{
Development of an Errorable Car-Following Driver Model
}

\author{
HSIN-HSIANG YANG*† and HUEI PENG $\dagger$
}

$\dagger$ Department of Mechanical Engineering, University of Michigan, Ann Arbor, MI 48109, USA

An errorable car-following driver model is presented in this paper. An errorable driver model is one that emulates human driver's functions and can generate both nominal (error-free) as well as devious (with error) behaviors. This model was developed for evaluation and design of active safety systems. The car-following data used for developing and validating the model was obtained from a large-scale naturalistic driving database. The stochastic car-following behavior was first analyzed and modeled as a random process. Three error-inducing behaviors were then introduced. First, human perceptual limitation was studied and implemented. Distraction due to non-driving tasks was then identified based on the statistic analysis of the driving data. Finally, time delay of human drivers was estimated through a recursive least square identification process. By including these three error-inducing behaviors, rear-end collisions with the lead vehicle could occur. The simulated crash rate was found to be similar but somewhat higher than that reported in traffic statistics.

Keywords: Active Safety, Car-Following, Driver Model, Driver Errors

\section{INTRODUCTION}

Driver behavior is an important topic for transportation and ground vehicle research. The way drivers respond to the surrounding traffic influences roadway designs, traffic rules and human-vehicle interface design. Driver inattention or distraction is responsible for $25 \%-30 \%$ of police-reported crashes [1]. Inattentive drivers were found to have three - six times higher near-crash/crash risk than drivers who are attentive [2]. Over the past decade, Active Safety Technology (AST) was developed to assist human drivers to avoid or mitigate accidents; in other words, they are useful when the driver is either making a mistake or simply is not able to handle the situation. To evaluate the effectiveness of AST, driver models that achieve driving tasks normally are not very useful. A new kind of driver models need to be developed based on the concept that a driver model that normally achieves driving tasks could be perturbed to make mistake like human drivers. When the error mechanisms and the parameters are properly 
designed, this type of driver models can generate crash or near-crash behaviors that are of interest to engineers who are developing AST. To our best knowledge, such "errorable" driver models have not been studied in the literatures. This main contribution of this paper is the development of an architecture and tuning process for errorable driver models.

The focus of this paper will be on the longitudinal car-following behavior, which is important because rear-end collisions account for about $60 \%$ of all crashes [3]. Rearend collision warning/collision avoidance system were developed to avoid or mitigate this type of accidents. The effectiveness of $\mathrm{CW} / \mathrm{CA}$ system working with a human driver or other human controlled vehicle needs to be evaluated exhaustively because of legal/liability and other concerns. Errorable driver models constructed and used properly, can capture human/control interaction and thus accelerate the $\mathrm{CW} / \mathrm{CA}$ system development process. To make a model errorable, mechanisms of driver errors and their effects on driving must be understood. Driver errors can be viewed as a recurring event which, when combined with events from surrounding vehicles, could result in an accident-but in a stochastic fashion. For example, a driver may be distracted or engaging in alter-control tasks and thus fails to adjust vehicle speed properly. If the leading vehicle happens to decelerate at a wrong moment, a rear-end collision could happen. The human behavior (distraction) and the lead vehicle deceleration can both be described by stochastic processes. If proper human cognition/error mechanisms are included and proper probability distribution functions are used to introduce human errors, it is possible to reproduce accident/incident behavior that is statistically similar to field testing results - which is the final goal of this paper.

\section{LONGITUDINAL DRIVER MODELS}

As early as the 1950's, car-following driver models were developed to evaluate traffic capacity and congestion. Pipes [4] suggested a linear follow-the-leader model, which assumes a linear relationship between drivers' desired acceleration and the speed difference between the following and leading vehicles. The desired acceleration is realized after a neuromuscular delay. The Proportional gain and neuromuscular delay of drivers were identified by Chandler [5]. Gazis [6] modified this linear follow-the-leader 
model with a nonlinear approach. The proportional gain of acceleration in Pipes' model was replaced by a function of the $m^{\text {th }}$ power of range and the $l^{\text {th }}$ power of the following vehicle speed. The parameters $m$ and $l$ were estimated by several researchers [7]-[9]. Newell [10] proposed another nonlinear model that the desired speed is an exponential function of range errors. All models above describe drivers' objective as either zero range error or zero range-rate. Intuitively, drivers are more likely to do both. This hypothesis was first discussed by Helly [11]. In Helly's model, the acceleration is a function of both range error and range-rate. Tyler [12] used the linear optimal control approach to approximate human driving behavior. A quadratic function of range error and range-rate was optimized and the resulting model has the same form as Helly's except that the desired range is only a function of the controlled vehicle speed. Later on, Burnham [13] included driver delay and nonlinear vehicle dynamics into Tyler's model. Other than following the lead vehicle, a safety distance strategy was proposed by Gipps [14]. By estimating the kinematic relationship between the lead vehicle and the following vehicle in braking situation, a safe-distance is calculated. A second mode was added in the Gipps' model to describe free flow condition. Switching between the two modes, this model can capture driver's behavior under different traffic flows. Bando [15] adopted a hyperbolic tangent function to mimic the switching mechanism. By doing so, a single mode model can be used to describe both free and congested traffic flow.

Other than measuring dynamic variables and modeling driver decision as a continuous dynamic process, psychophysical studies provided an alternative for modeling car-following behaviors. Michael [16] argued that drivers would detect range variation through the change of the lead vehicle size. Once the change exceeded a threshold, the driver would adjust vehicle acceleration until a proper perceptual size was regained. The driver's behavior was modeled as a sequential control, which responds to an event (exceeding threshold) and resets the action when a new event is triggered. Some basic concepts and criteria were provided in Michael's work but no specific mathematic model was presented. Lee [17] claimed that drivers use time-to-collision as the perceptual threshold for braking and used $\tau$ (angular separation over separation rate) to estimate time-to-collision. In Lee's hypothesis, the driver initiates brake action based on $\tau$ threshold and controls the brake force based on the time rate of change of $\tau$. This 
hypothesis was verified by Yilmaz [18] but a general agreement about the threshold value is not reached. Reiter [19] used range as the perceptual threshold and provided experimental results to obtain model parameters. On the other hand, Brackstone [20] conducted experiments based on a similar approach, but he uses range-rate instead of $\tau$.

From the literature above, it can be seen that both engineering and psychophysical approaches were attempted to model driver behaviors. Engineering models focused on the physical signals instead of what happened internal of the driver. Psychophysical models help to qualitatively explain the driver's actions. In this paper, an engineering type of longitudinal driver model will be used as the basis of this errorable model to perform normal car-following behavior. Psychophysical approach will be used to introduce error mechanisms. The parameters of the error mechanisms will be obtained through naturalistic driving data.

\section{ERRORABLE CAR-FOLLOWING DRIVER MODEL TEMPLATE}

To develop an errorable model, a model that normally achieves car-following tasks will be used as a template. Many existing car-following models can fulfill this need. The majority of those models assume driving as a deterministic process, i.e., the vehicle states can be calculated exactly from dynamic equations [12], [14] or heuristic rules [19], [20]. Precise prediction of vehicle states might be useful in traffic flow analysis, but has little use for AST development. In actual driving, human do not behave deterministically and some randomness is always observed. This stochastic behavior of driving has been studied in [22]-[25]. Human randomness is commonly modeled as a random noise. By adjusting magnitude and parameters of the noise, the driver model can be tuned to fit the test data. However, the tuning processes of noise are not convincing and have little reflection of actual driving behavior. In this section, stochastic behaviors of human drivers will be analyzed with more physical insights. Hypothesis will be validated with naturalistic driving data and a stochastic driver model will be development. This model should capture human's normal driving behaviors as well as some deviations. Those deviations are considered as part of driver's nature, not error. The resulting normal and deviated behaviors should be consistent with what we observed in realistic driving data in a statistic fashion. In Section 4, the stochastic driver model 
will combine with error mechanisms derived from driving data; then, this new model can be made to make mistake in a controlled fashion, in other words, errorable.

\subsection{Stochastic Behavior of Longitudinal Driving}

During a car-following task, the driver normally regulates the vehicle speed so that the space between the following vehicle and the leading vehicle is at a desirable level, even under lead vehicle speed fluctuation. This process is usually modeled by a deterministic process [1]-[6], with the desired velocity calculated from kinematic or dynamic equations. It might be a good approximation for traffic analysis; however, it is not suitable to describe individual driver behavior, because human cannot perceive, calculate, or control perfectly and human is also unlikely to perform such tasks at a regular tempo.

\subsubsection{Hypothesis}

In everyday driving, human drivers normally aim to regulate the vehicle speed so that the spaces between the following vehicle and the leading vehicle are large enough. The above statement has two assumptions: human drivers have intention to achieve a desired vehicle state (speed) and as long as this state was roughly achieved, some deviations would be acceptable, and unavoidable. The deviation of control is due to various reasons including driver's imperfection in perception, control, or exogenous disturbances (powertrain dynamics, road gradient, etc.) The assumption regarding deviation of control was discussed in [25] and modeled as a random noise. However, the color and magnitude of the stochastic process are unknown and a tuning step is needed. In [26], the deviation of braking control of human drivers under deceleration was modeled as a truncated Gaussian distribution. In this study, the deviation will be modeled and assumed to exist in both braking and accelerating, and the distribution will be obtained from naturalistic driving data.

\subsubsection{Modeling of Stochastic Behavior}

In this section, drivers are assumed to have a target acceleration which is a function of range, range-rate, and/or time headway. 


$$
a_{d}(t)=f_{a_{d}}\left(R(t), \dot{R}(t), T_{h}\right)
$$

Unlike most of the existing models, this desired state will not be achieved exactly and, instead, will have some uncertainties dependent on range. When the range is large, the driver cannot perceive range accurately and there is more room to deviate. Thus, the deviation increases with range. This hypothesis is summarized below and will be verified in the following section.

$$
\sigma(t) \propto f_{\sigma}(R(t))
$$

\subsection{Data Analysis and Validation}

The hypothesized model in Eqs. (3.1) and (3.2) was verified with field test data, which are from Road-Departure Crash-Warning System Field Operational Test [27]. The RDCW FOT was designed to study road departure behavior. This system was implemented on 11 passenger vehicles with data acquisition systems. Seventy-eight test drivers participated and each drove a test vehicle for four weeks. Total data set accumulated 83,000 miles of driving and over 400 signals were captured at $10 \mathrm{~Hz}$ including signals useful for our study, such as range, range-rate, following vehicle acceleration, etc. Participants were instructed for road departure field operational test; no restriction what-so-ever in longitudinal driving. Therefore, this field test data is naturalistic in car-following and ideal for analyzing human driver's stochastic behaviors.

\subsubsection{Range-rate and Acceleration Distribution}

In Eg. (3.1), the desired acceleration was assumed to be a function of range, range-rate, and/or time headway. First, we started from a simple linear relationship (3.3) which is inspired by the Pipe's model [1].

$$
a_{d}(t)=P \cdot \dot{R}(t)
$$

It can be seen from Figure 3.1 that this simple relationship does not describe the test data very well. However, with the proportional gain $P$ in Eg. (3.3) replaced by a function of range, the data fit quality is much improved (see Figure 3.2). 


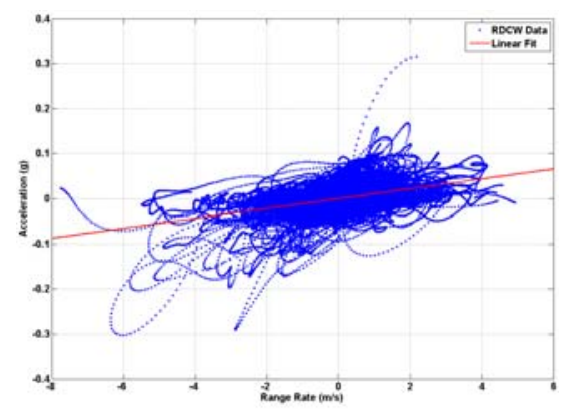

Figure 3.1 RDCW data acceleration vs. range-rate
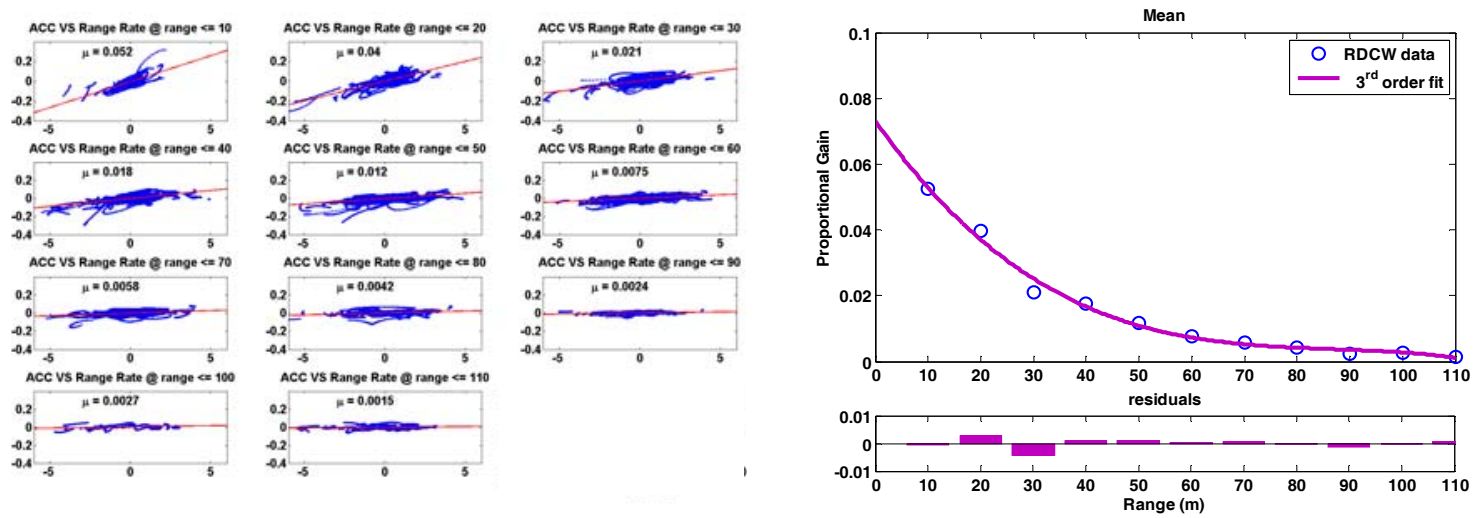

Figure 3.2 RDCW data acceleration vs. range-rate with various range

The sensitivity function $P(R(t))$ was found to be a cubic function of $R(t)$, i.e.

$$
\begin{gathered}
a_{d}(t)=P(R(t)) \cdot \dot{R}(t) \\
P(R(t))=P_{3} \cdot R^{3}(t)+P_{2} \cdot R^{2}(t)+P_{1} \cdot R(t)+P_{0}
\end{gathered}
$$

Figure 3.2 shows that the accelerations of the following vehicle are proportional to the range-rate, but with a small scatter around the linear fit. The distributions and the residues were plotted in Figure 3.3 with their standard deviations calculated. The standard deviation can be fitted by a second order polynomial reasonably well. 

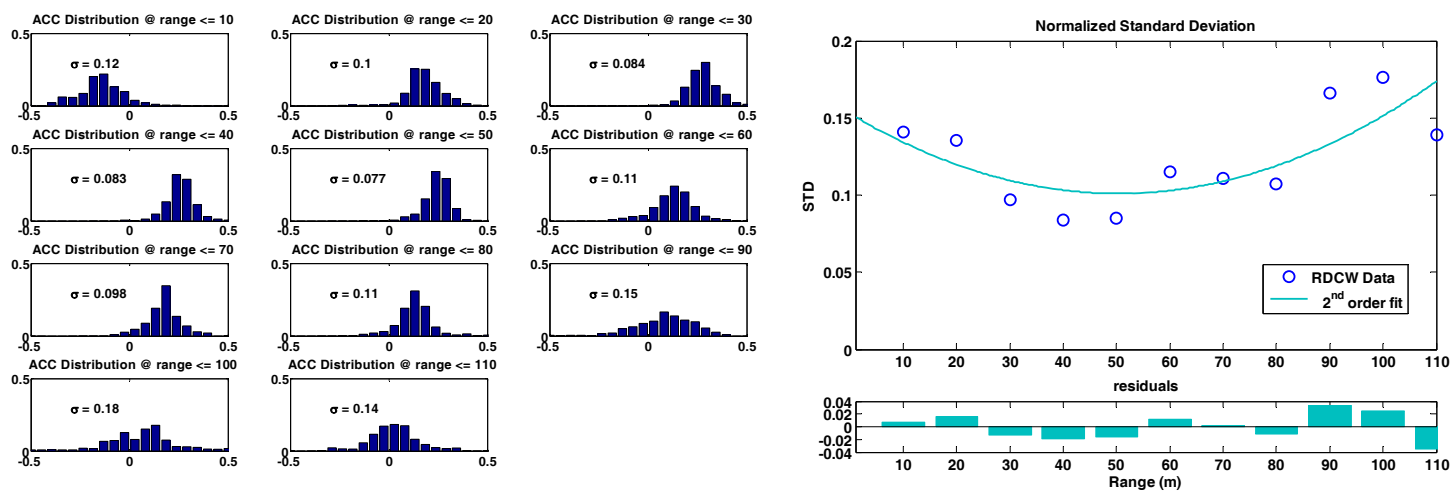

Figure 3.3 RDCW data acceleration distribution with various range

$$
\sigma(R(t))=\bar{P}_{2} \cdot R^{2}(t)+\bar{P}_{1} \cdot R(t)+\bar{P}_{0}
$$

The vehicle acceleration under human driver control is generated from a stochastic process with a mean of $a_{d}(R(t))$ and standard deviation $\sigma(R(t))$. The overall stochastic driver model is summarized below.

$$
\begin{gathered}
a_{d}(t)=P(R(t)) \cdot[\dot{R}(t)] \\
P(R(t))=P_{3} \cdot R^{3}(t)+P_{2} \cdot R^{2}(t)+P_{1} \cdot R(t)+P_{0} \\
\sigma(R(t))=\bar{P}_{2} \cdot R^{2}(t)+\bar{P}_{1} \cdot R(t)+\bar{P}_{0} \\
a(t)=f\left(a_{d}, \sigma\right)
\end{gathered}
$$

A remaining unknown in the stochastic driver model is the density function $f$ in Eq. (3.7). Brunson [26] adopted a truncated Gaussian distribution for the decelerating cases. However, the observation in Figure 3.3 shows that the distribution is not symmetric. Lognormal distribution (3.8) or extreme value (3.9) distribution [28] are two possible candidates for non-symmetric distributions.

$$
\begin{gathered}
f_{\operatorname{logn}}(x \mid \mu, \sigma)=\frac{1}{x \cdot \sigma \cdot \sqrt{2 \pi}} \cdot \exp \left(\frac{-(\ln x-\mu)^{2}}{2 \sigma^{2}}\right) \\
f_{e v}(x \mid \mu, \sigma)=\sigma^{-1} \cdot \exp \left(\frac{x-\mu}{\sigma}\right) \cdot \exp \left(-\exp \left(\frac{x-\mu}{\sigma}\right)\right)
\end{gathered}
$$

Simulation results show that the extreme value distribution has better agreement with the RDCW data. Therefore, Eq. (3.9) will be used in this study. One fitting example with range between 40-50 m was showed in Figure 3.4. 




Figure 3.4 Distribution fitting of the RDCW acceleration data

\subsection{Extension for Range Regulation}

In Eq. (3.4), the driver only responds to non-zero range-rate. Therefore, the model may be trapped in a tailgating situation; in other word, the model may follow the lead vehicle with a very small range. Moreover, the proportional gain in Eq.(3.5) is a function of range. If the range is too large (e.g. $>70)$, the model will hardly response to any stimulus; the following vehicle will thus fall behind. Both situations are considered possible but not normal car-following behavior.

\subsubsection{Range Regulation}

In reality, human drivers regulate range or time headway in addition to rangerate [11][12]. Human driver are assumed to have a desired range and will regulate the vehicle speed to achieve the desired range. To capture this behavior, an extra term was included in Eq. (3.4).

$$
a_{d}(t)=P(R(t)) \cdot \dot{R}(t)+C \cdot\left(R(t)-T_{h} \cdot V_{F}(t)\right)
$$

where $\mathrm{C}$ is a constant gain for range regulation and $T_{h}$ is the time headway.

\subsubsection{Time Headway}

The time headway $T_{h}$ in Eq. (3.10) was obtained from steady-state cruising RDCW data. The actual time headway of each human driver can be calculated and a example distribution was shown in Figure 3.5. 


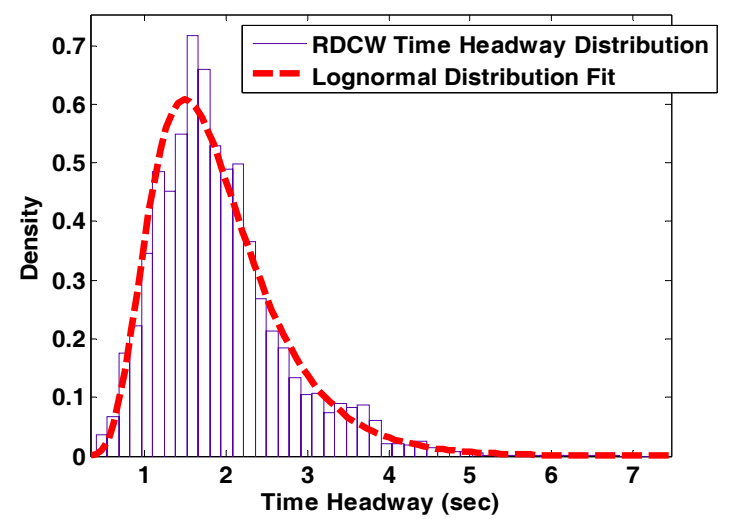

Figure 3.5 Time headway distribution and fitting results

The time headway distribution is approximated as a random process with lognormal distribution. However, this random process generates a random variable every sampling time. In real driving, human drivers obviously do not adjust their time headway so frequently. By adding a 4-step running average filter, the output becomes smoother and similar to actual driving data.

\subsubsection{Driver Model Diagram}

A schematic diagram of the stochastic driver model is shown in Figure 3.6. The inputs are the desired time headway $\left(T_{h}\right)$ and the lead vehicle velocity $\left(V_{L}\right)$, which is from naturalistic driving data. The average desired acceleration $\left(a_{d}\right)$ and deviation $(\sigma)$ are calculated from Eq. (3.10) and Eq. (3.6), respectively. The actual acceleration is then generated through a random number generator (MATLAB function "evrnd") and the output $V_{F}$ can be integrated. This model simulates the normal driver behavior and random deviations derived from actual driving data. Three different simulations (under the same lead vehicle stimulus) are shown in Figure 3.7 to illustrate the stochastic natural of this model. 


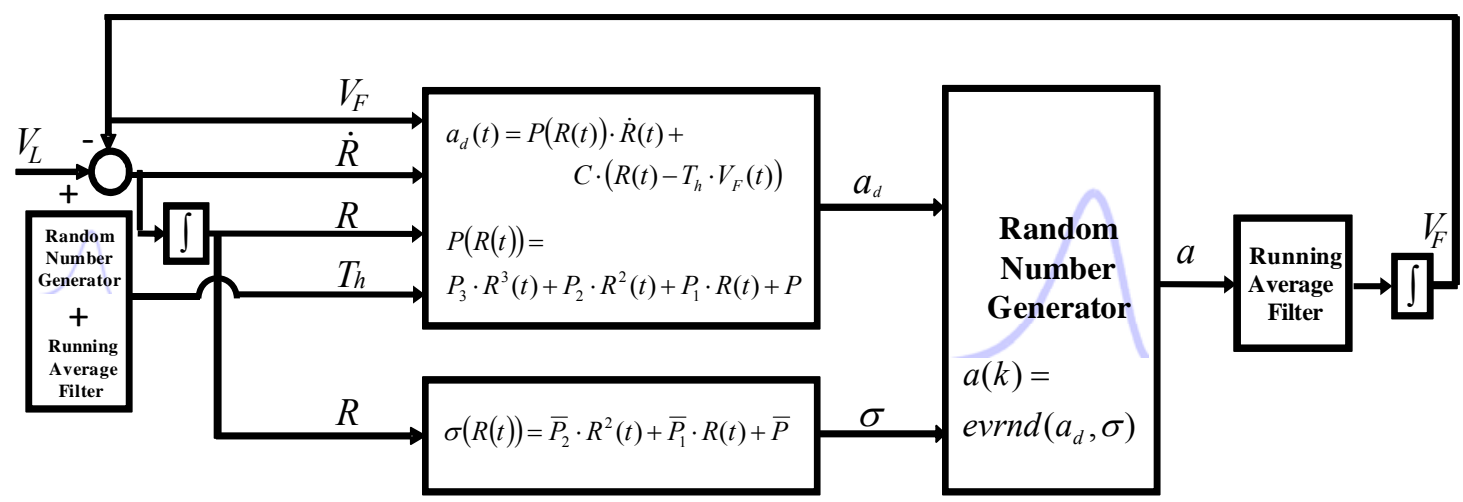

Figure 3.6 Stochastic driver model diagram


Figure 3.7 Examples of stochastic driver model simulation

\subsection{Simulation Results and Discussion}

The proposed stochastic driver model (SDM) was evaluated by the RDCW data. The lead vehicle speed from the RDCW data is used as the input to the SDM. The SDM response is compared with the measured following vehicle speed from the RDCW database. The model performance is assessed by examining the model prediction error. In addition, the SDM performance was also assessed by comparing its range-range rateacceleration behavior with other driver models.

\subsubsection{Evaluation by RDCW}

In the RDCW database, driving records of 78 drivers' data are available. Based on the query criteria, the car-following data was extracted. The obtained query results include between 20 and 74 maneuvers from each driver. Those maneuvers were 
separated into a training set and an evaluating set. The ten longest maneuvers of each driver were used as the training data for calibrating the SDM parameters. Then, the next ten longest maneuvers were used as the evaluating sets. The measured lead vehicle speed is used as the input and the model predicted $V_{F}$ is compared with the experiment data from the RDCW. The model prediction error distributions of 10 randomly selected drivers were shown in Figure 3.8.

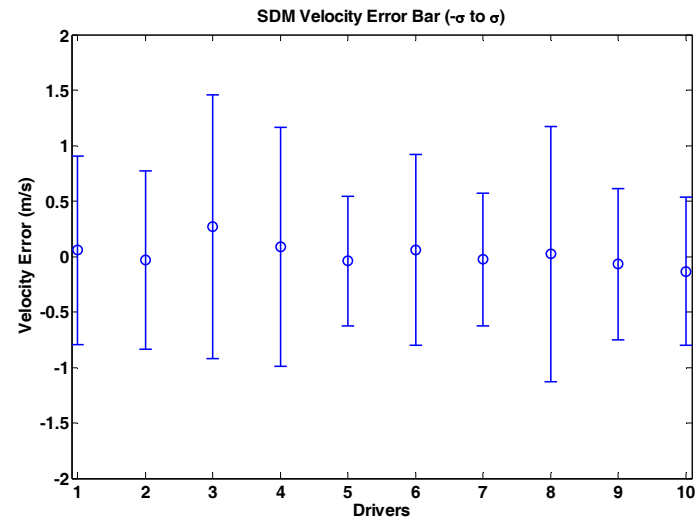

Figure 3.8 Distribution of model prediction error

To assess the performance of the stochastic longitudinal driver model, the performance of several deterministic longitudinal driver models is also studied. Three models were selected because of their simplicity and accuracy. Note that, Lee [21] reported that the Gipps model [14] has the best performance for fitting human driver in his study. However, the Gipps model generates a desired velocity. When applying parameters to evaluating sets, this desired speed generated from training sets would not be valid. Therefore, the Gipps model was not selected as a model to compare with.

The same training sets used to obtain the SDM parameters were selected and a numerical optimization technique was applied to obtain the best parameters for the three models. For each driver, there are ten maneuvers in the training sets; hence ten sets of parameters were obtained. The parameter values were averaged and applied to the evaluating maneuvers. The distributions of the velocity error are shown in Figure 3.9. 




Figure 3.9 Model comparison results

For the training set alone, the SDM has the worst performance in terms of standard deviation of the predicted speed. However, several facts should be pointed out:

- SDM did not go through an optimization scheme for each training maneuver.

- SDM used only one set of parameters for all ten maneuvers in the training set, meanwhile all other models used a unique parameter set for each maneuver.

For the evaluation set, the SDM has the most consistent performance among all models. The error of the SDM increases only slightly while all other models' performances degrade significantly. This indicts that deterministic driver models with optimized parameters can fit human driver behaviors well but are not robust in prediction.

Another significant advantage of SDM is demonstrated by examining the acceleration and range-rate plots (Figure 3.10). The acceleration, range and range-rate distributions of the SDM were plotted in Figure 3.10 to compare with the RDCW data qualitatively. 

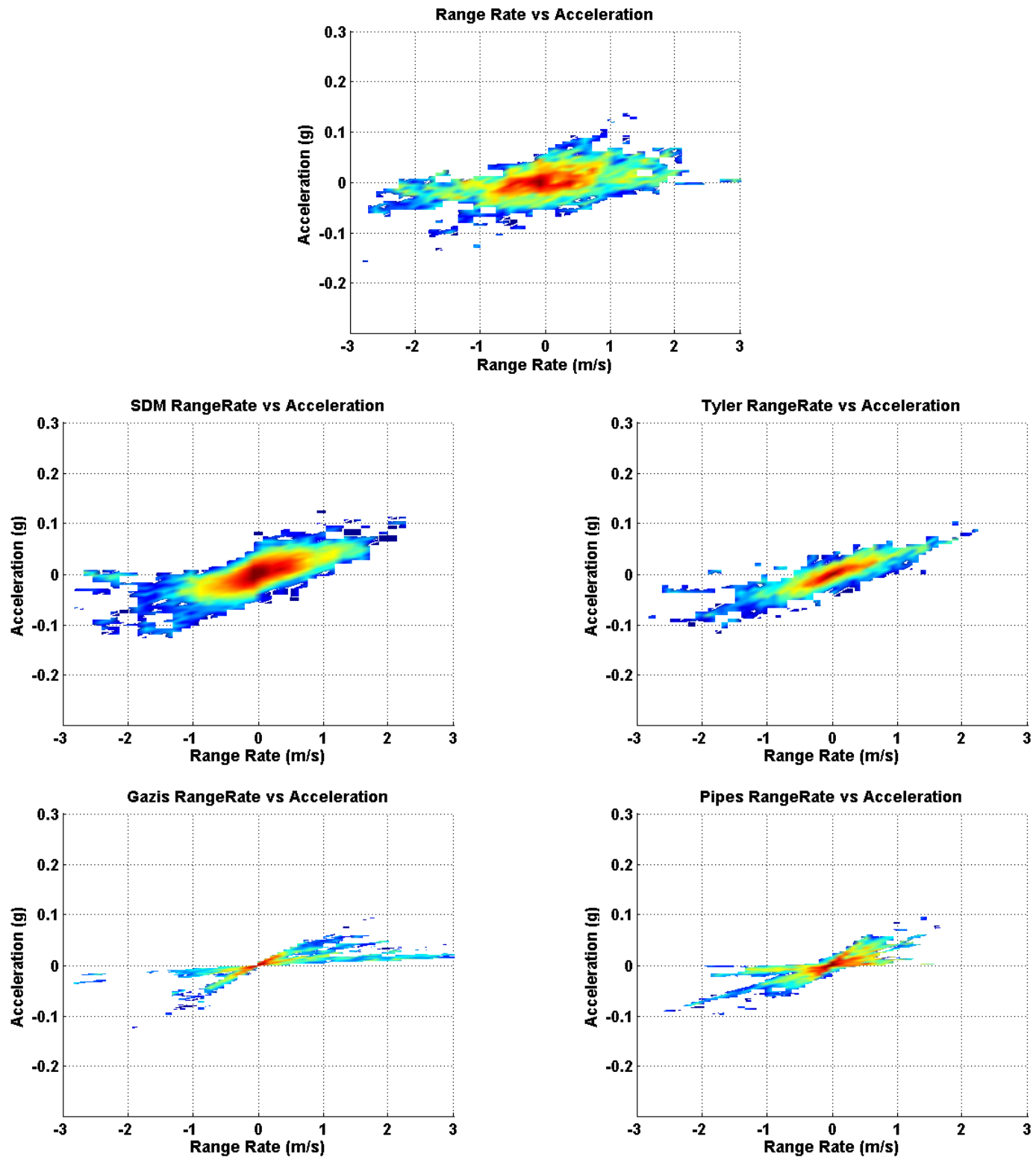

Figure 3.10 Range and range-rate distribution comparison

It can be seen that except the SDM and the Tyler's model, all other models have very different data distribution than observed in the RDCW data. Because of the tight regulation of time headway, Tyler's model has an elliptic shape distribution similar to RDCW. However, its distribution still did not spread out enough. This is because these deterministic models are "optimal" models and do not capture the stochastic behavior of drivers adequately. 


\subsection{Discussion}

A stochastic longitudinal driver model was developed and evaluated against naturalistic driving data. Sufficient flexibility was shown in training this model to fit experimental data. Meanwhile, superior robustness was demonstrated in predicting the human driving behavior in the evaluation test sets. This SDM captures human's normal driving behaviors with deviations similar to actual driving data.

The SDM will serve as driver model template for the development of the errorable driver model, which is the focus of the rest of the paper. Combining with error mechanisms derived from human behavior, this model can be manipulated to make mistake, i.e., errorable. To model driver error mechanisms, driver errors and their effects on driving must be understood. In the following section, three human driving error mechanisms will be studied and implemented in the SDM.

\section{LONGITUDINAL ERRORABLE CAR-FOLLOWING DRIVER MODEL}

The final goal of this paper is to build a longitudinal car-following driver model for evaluating active safety technology (AST) systems. Models that achieve driving tasks perfectly are not useful. On the contrary, a model that makes mistakes similar to human drivers is useful for the development of AST. We believe a car-following driver model can be manipulated to make mistakes by introducing proper error mechanisms. The analysis and modeling of error mechanisms will be discussed in this section and the resulting errorable driver model is validated against actual traffic data.

\subsection{Error-Inducing Behaviors}

Three types of error-inducing behaviors are analyzed: perceptual limitation, time delay, and distraction. Each of them degrades the car-following performance separately. The error-inducing behaviors are modeled as stochastic processes based on their frequency of occurrence. Then, these stochastic processes are introduced into the SDM independently. 


\subsubsection{Perceptual Limitation}

Human drivers sense and perceive environmental variables such as range, range rate and vehicle speed to perform the driving task. Among all the feedback information human drivers perceive, vision is the primary source when operating motor vehicles [31][32]. In a car-following task, the abilities to detect changes in distance and velocity are critical. Therefore, they need to be studied as one of the error-inducing behavior in this research.

The proposed SDM uses range and range-rate as two feedback cues. The range between vehicles is perceived though optical invariants [33]. The perception is then limited by the resolution of human vision system. A typical accepted localization threshold is $6 \operatorname{arc} \sec (0.5 \mu$ on the retina). Other than visual angle, human also utilizes environmental information such as eye-height, relative position, and texture of the ground [34]. Therefore, we do not implement the perceptual limitation of distance or range.

How human drivers perceive relative speed has long been a research pursuit. Many researches were done aiming to answer the following question: Can human perceive relative speed directly or is it indirectly inferred through the change in position? This question is sometimes recast in a different form: is perceptual sensitivity of velocity different from sensitivity of position? Nakayama [35] used a random-dot pattern movement to isolate the motion from position cues. Randomly generated dot pattern prevented the position comparison and allow motion detection to be the only mechanism. The result concluded that motion and position perception are two different mechanisms. In the low frequency range $(<2 \mathrm{~Hz})$, human has a direct assessment of speed. But for higher frequency $(>2 \mathrm{~Hz}$ ), position derivative seems to dominate. A different perceptual threshold was also reported. Various Just-Noticeable Differences $(\Delta V / V)$ of velocity discrimination from 0.05 to 0.2 were found in [36]-[39]. Since velocity detection in a carfollowing task is relatively easy (comparing to other tasks such as hitting a baseball); a velocity threshold of 0.1 is used in this paper.

The perceptual limitation was simulated through quantization for the range-rate input. It is implemented as follows: 


$$
\dot{R}^{\prime}(k)= \begin{cases}\dot{R}(k) & \text { if } \frac{\dot{R}(k)-\dot{R}^{\prime}(k-1)}{\dot{R}^{\prime}(k-1)} \geq 0.1 \\ \dot{R}^{\prime}(k-1) & \text { otherwise }\end{cases}
$$

, where $\dot{R}$ is the actual range-rate and $\dot{R}^{\prime}$ is the perceived range-rate. An example simulation for the perceptual limitation quantizer was shown in Figure 4.1.

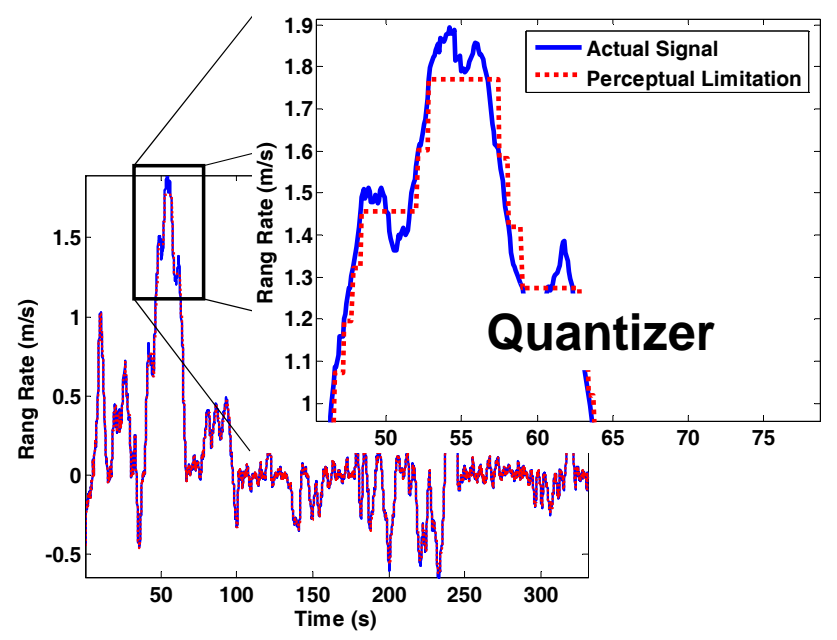

Figure 4.1 Range rate perceptual limitation simulation

\subsubsection{Time Delay}

Time delay is another source of error. Neuromuscular delay and brain processing time are two major sources of time delay. Neuromuscular delay may be close to constant for each driver [45], but the brain processing time is not. Therefore, the total time delay is varying. The truth is that the delay time may very well be related to distraction due to secondary tasks. However, it is important to include delay as a separate error mechanism in the SDM. A parallel recursive least square (RLS) method was used to estimate the varying total time delay. Thirty ARMA models with different delay steps (1 to 30) were used to fit the test data simultaneously by the RLS algorithm. For every single data point, the delay step of the most accurate ARMA model was chosen as driver's time delay, and then the time delay sequence can be constructed. The driver time delay was reported to be $.5-.9 \mathrm{sec}$ from an instrumented vehicle in a test track [47] responding to a signal change. A longer brake response time was found (1.5-3.5 sec) in a driving simulator [48] for the car-following and in normal traffic (3.5-4.5 sec) [49]. The value for $\mathrm{N}$ is selected 
to be thirty (three second). The estimated results of the RDCW data were analyzed. One example was shown in Figure 4.2.
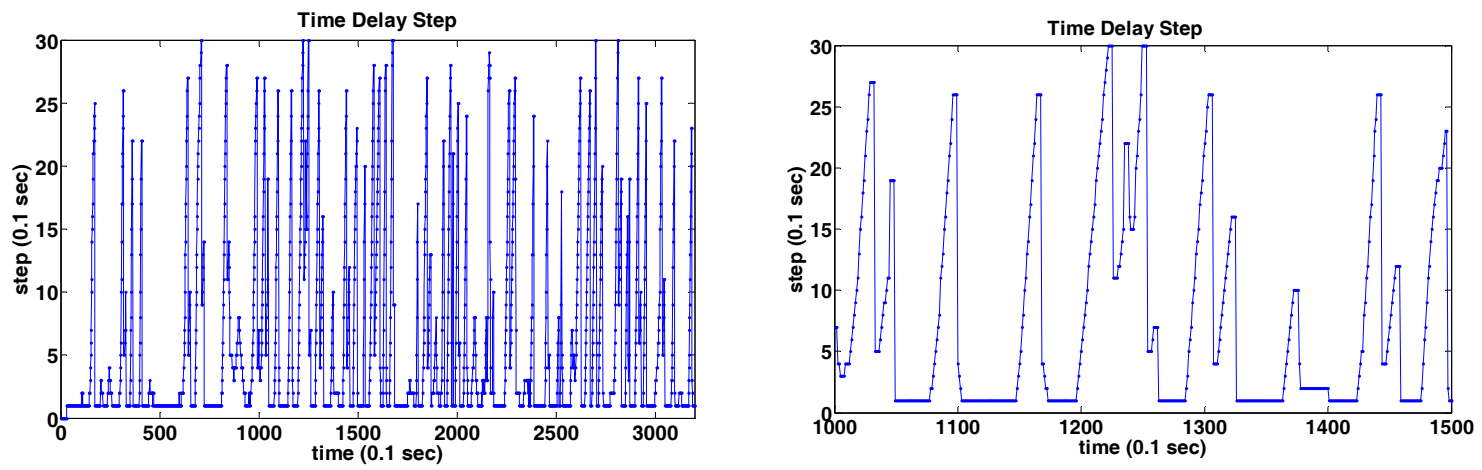

Figure 4.2 Time delay estimation of RDCW data

This sequence shows a significant character that the delay step increases with time and, then dropped or reset to zero. This can be modeled by a renewal process as well.

To duplicate the characteristic of time delay observed in the previous section, a probability distribution of time delay was first obtained from the time delay sequence. If time delay step is zero, we define it as normal driving and their distribution was showed in Figure 4.3 (a) which can be fitted by an exponential distribution. When time delay step increased from zero, we define it as delayed driving and their distribution can be approximated as uniform (Figure 4.3 (b)).

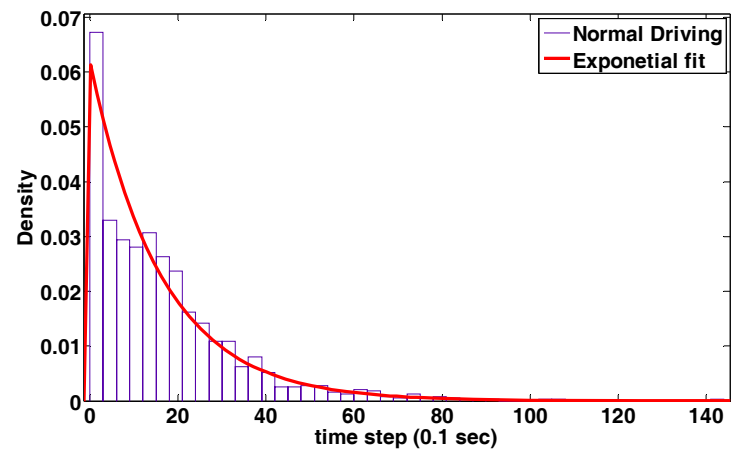

(a) Normal driving



(b) Delayed driving

Figure 4.3 Time delay distribution and PDF fitting

Next, the human time delay was modeled by a renewal process which has an inter-arrival time indicated by a probability density function. When a renewal arrives, the 
time delay step will increase from zero and reset until the next renewal. The resulting time delay sequence can represent the real sequence obtained by RLS and be used for simulations (Figure 4.4).
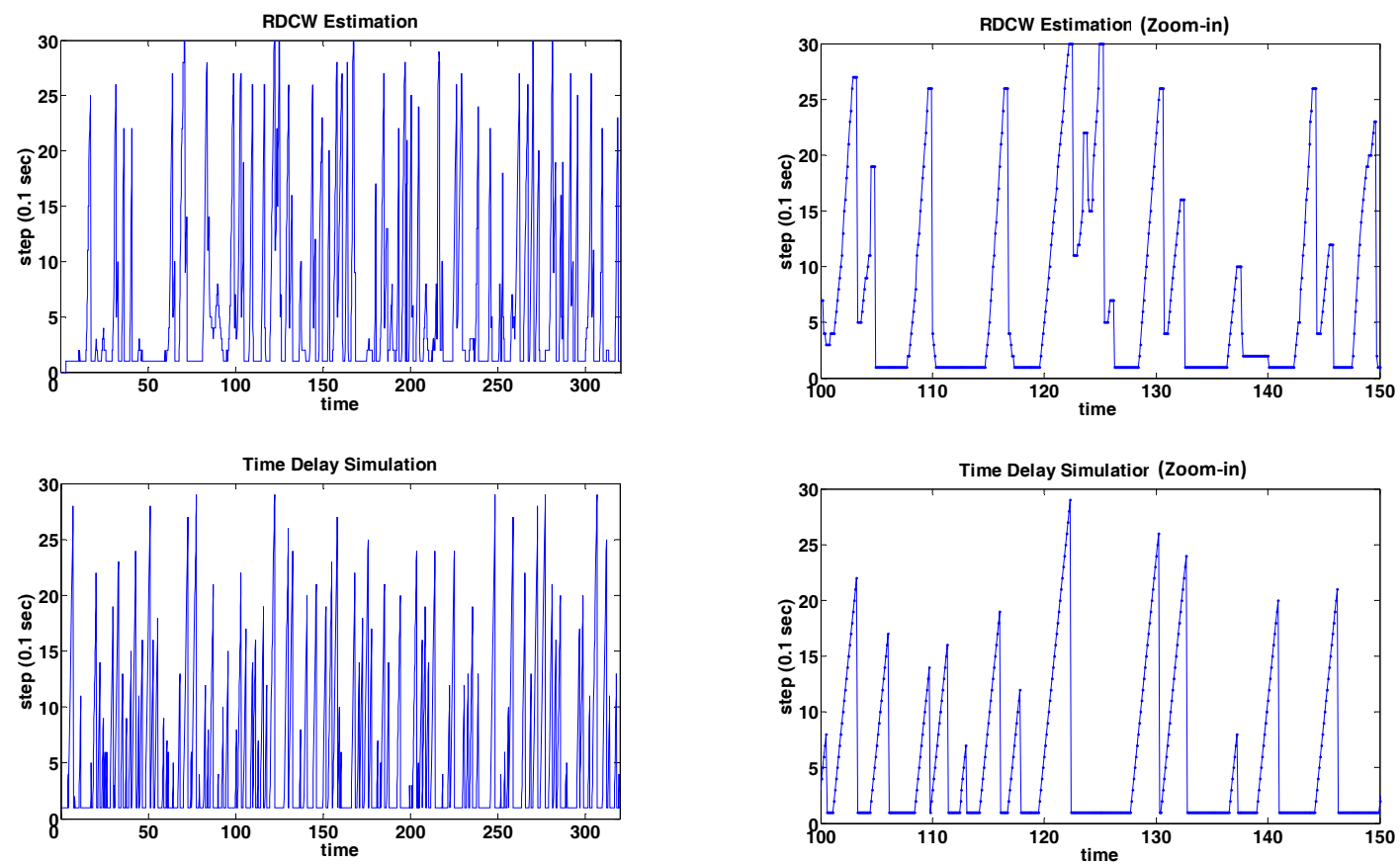

Figure 4.4 Time delay simulation and comparison with the RDCW estimation

\subsubsection{Distraction}

Driver distraction is responsible for almost thirty percent of police-reported crashes [1]. Therefore, it is an important error-inducing mechanism that should be included. Driver can be distracted by in-vehicle tasks including cell phone usage, or interaction with other passengers [41]. When a secondary task happens, the human driver will have reduced attention to driving. Some of the secondary tasks may require higher participation and may temporarily disable human driver's ability of to attend to driving (Figure 4.5). Sheridan [42] suggested a switching type of control configuration which can be used to model the transition between different modes of driving. In this paper, we define the multi-task driving as cause for "mind off the road". The human driver is assumed to keep his/her eyes on the road while doing the secondary task. However, the non-driving task increases the mental load and degrades the driving performance. Boer 
[43] reported an increasing deviation of steering wheel angle under mental load for lane keeping driving. The same increasing deviation of control should exist in the carfollowing driving. Meanwhile, if a human driver is fully engaged in a secondary task, very often he/she will move the eyes to this task and stop updating the driving information. We define this situation as "eyes off the road".

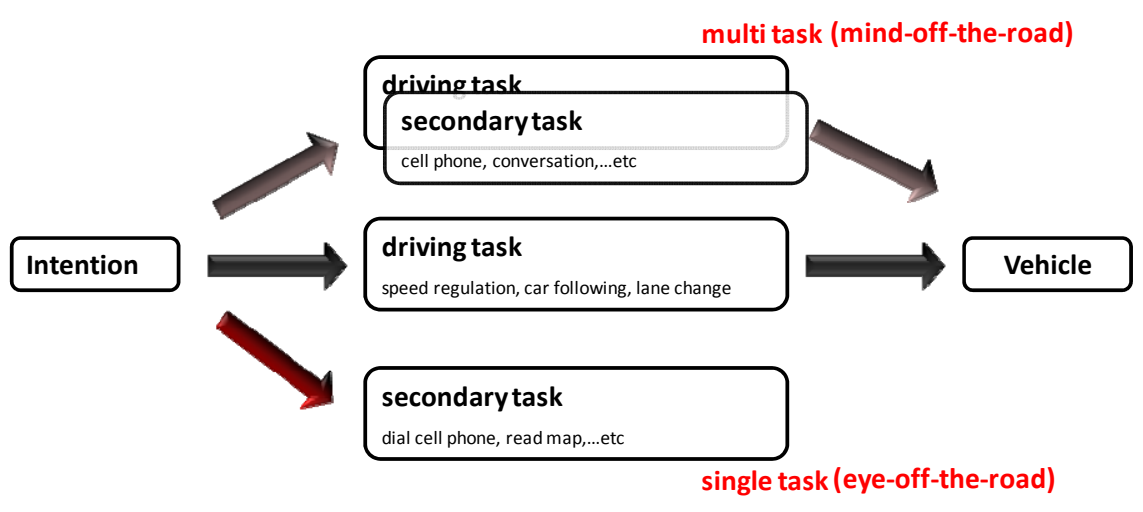

Figure 4.5 Driving under distraction

\subsubsection{Mind-off-the-road}

One challenge in the errorable driver model is to identify the "mind off the road" mechanism and quantify it for modeling. Driving data of 10 drivers was collected from the RDCW database. Based on the actual test data, the SDM can predict the next vehicle states and calculate their deviations respectively. If the test data landed outside one standard deviation of the prediction, we define it as a deviated behavior (Figure 4.6). In this paper, the deviated behavior was assumed to be a consequence of mind off the road.



Figure 4.6 Distraction prediction based on the SDM

This analysis was applied to the RDCW data and the results are shown in Figure 4.7. Once the RDCW $V_{F}$ lands within one standard deviation of SDM prediction, it will 
be defined as normal driving (the first two data points showed in left hand side of Figure 4.7). If the RDCW $V_{F}$ lands outside one standard deviation (the last two points in left hand side of Figure 4.7), it will be defined as deviated behavior. The predicted result was showed in right hand side of Figure 4.7 with dotted line and can be modeled by an alternative renewal process with two independent identical distributions (IID) (Figure 4.8).
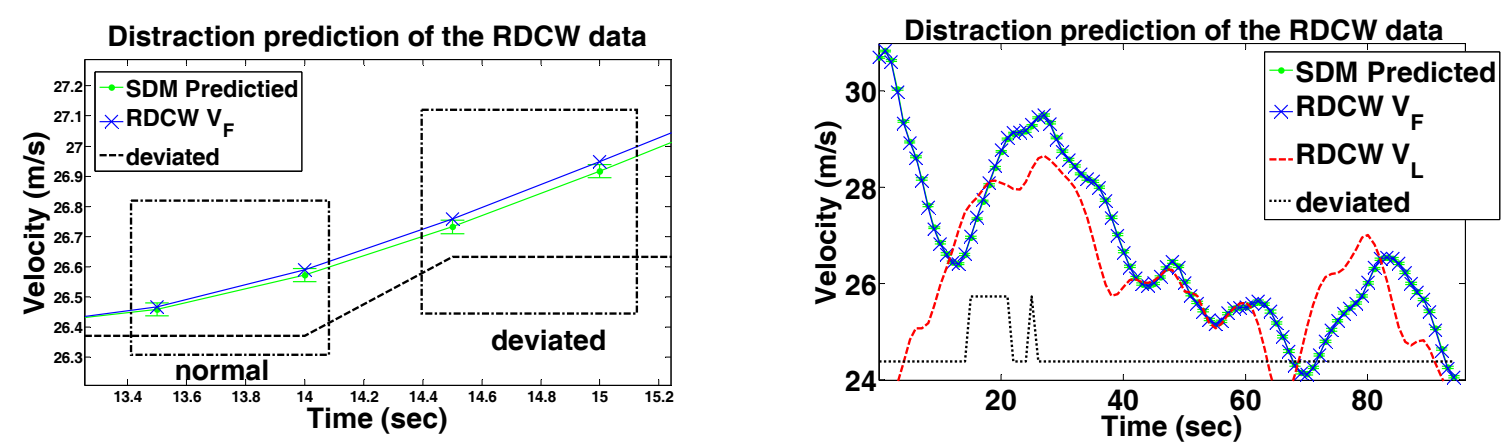

Figure 4.7 Distraction prediction of the RDCW data
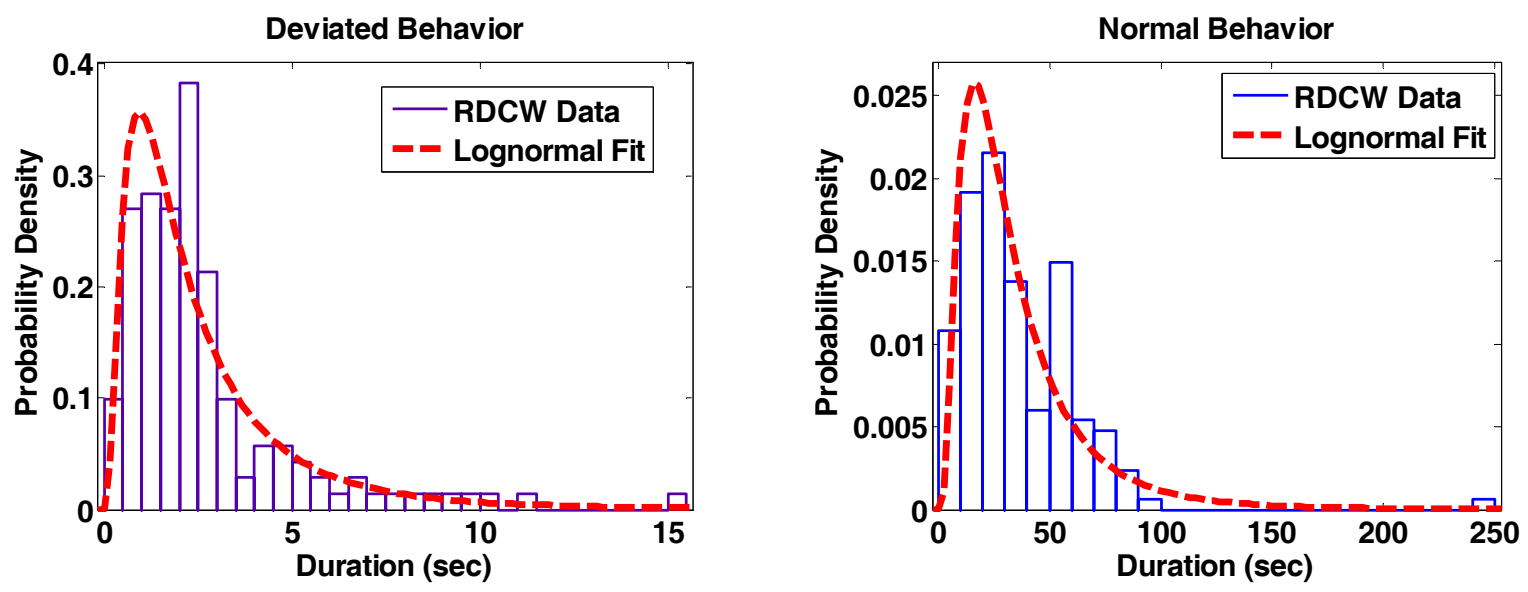

Figure 4.8 Distribution of the normal and deviated behavior

Every time the process has a renewal event which indicates the driver is distracted the deviation in the random number generator Eq. (3.7) will be increased to model the increase workload and the degraded control.

Table 4.1 Distributions parameters of mind-of-the-road.

Lognormal

Distribution

Mean (sec)

STD 


\begin{tabular}{ccc}
\hline Normal & 26.62 & 0.75156 \\
Deviated & 1.8465 & 0.89344 \\
\hline
\end{tabular}

The type of distraction was modeled as alternative renewal processes. Drivers are assumed to start at normal condition without distraction. After an inter-arrival time, which has mean and standard deviation listed in Table 4.1, drivers will become distracted for a short period of time. This process will be repeated through simulations and one simulation was illustrated in Figure 4.9.

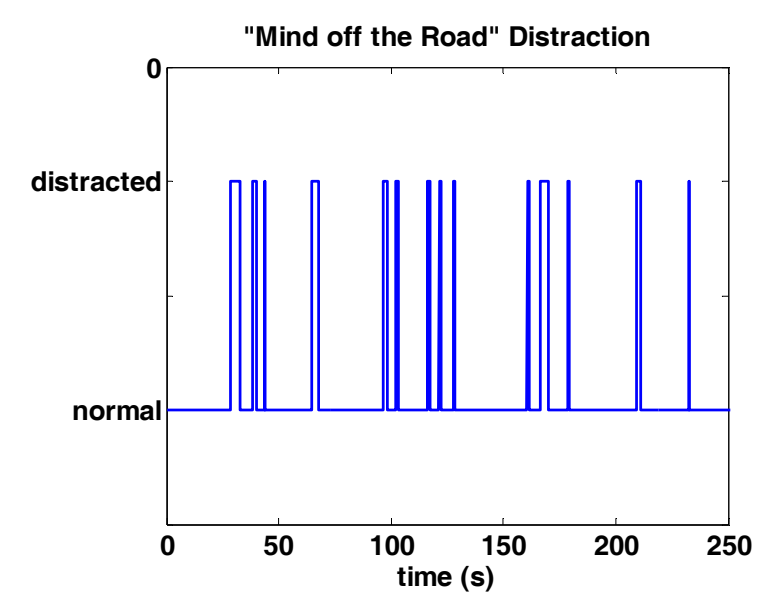

Figure 4.9 Example of renewal processes for the mind-off-the-road distraction

\subsubsection{Eyes-off-the-road}

When eyes are taken off the road, drivers stop updating the feedback information. The perceived range, range-rate, and speed would remain unchanged from the previous step. Drivers are also assumed to freeze their control actions at the previous level. Once the distraction ends, the driver will resume updating the information and perform proper control adjustment. The above statement can be realized by using a switch and a register (Figure 4.10). 


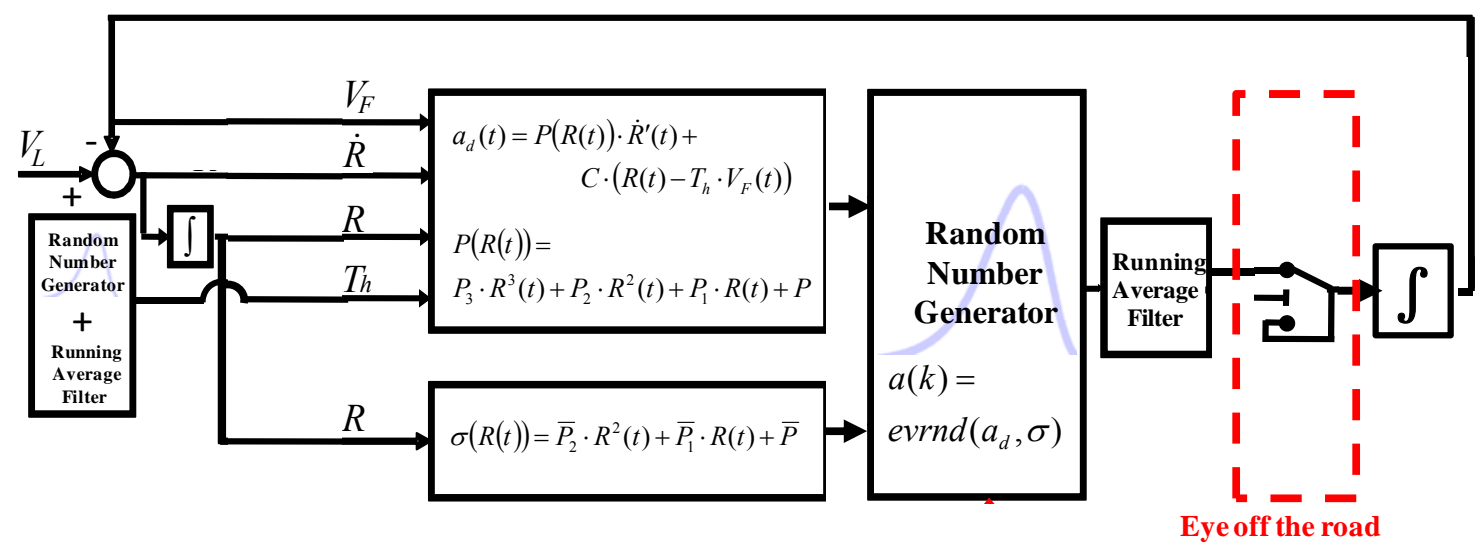

Figure 4.10 Block diagram with eyes off the road distraction module

The eye-off-the road behaviors were studied in several literatures and frequency of occurrences can be found in [1] and [46]. However, the effect of eye-off-the-road was already included in this errorable driver model when the time delay was modeled in section 4.1.2. In Figure 4.11, the time delay increased linearly with time. In other words, the driver model keeps using the same old data without updating, which is equivalent to switch off the information flow. Therefore, the additional switch mechanism will not be implemented in order to avoid over-estimation of eye-off-the-road.

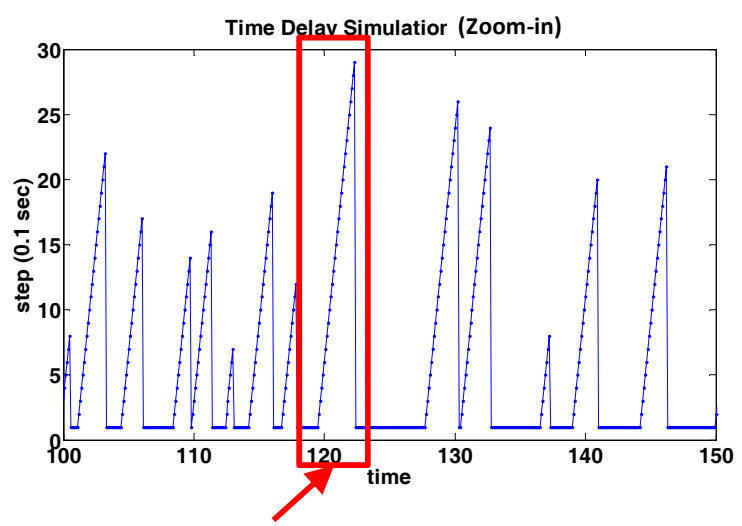

Eye-off-the-road

Figure 4.11 eye-off-the road modeling

\subsection{Simulation Results}

The final errorable driver model including the three error-inducing mechanisms is shown in Figure 4.12. 


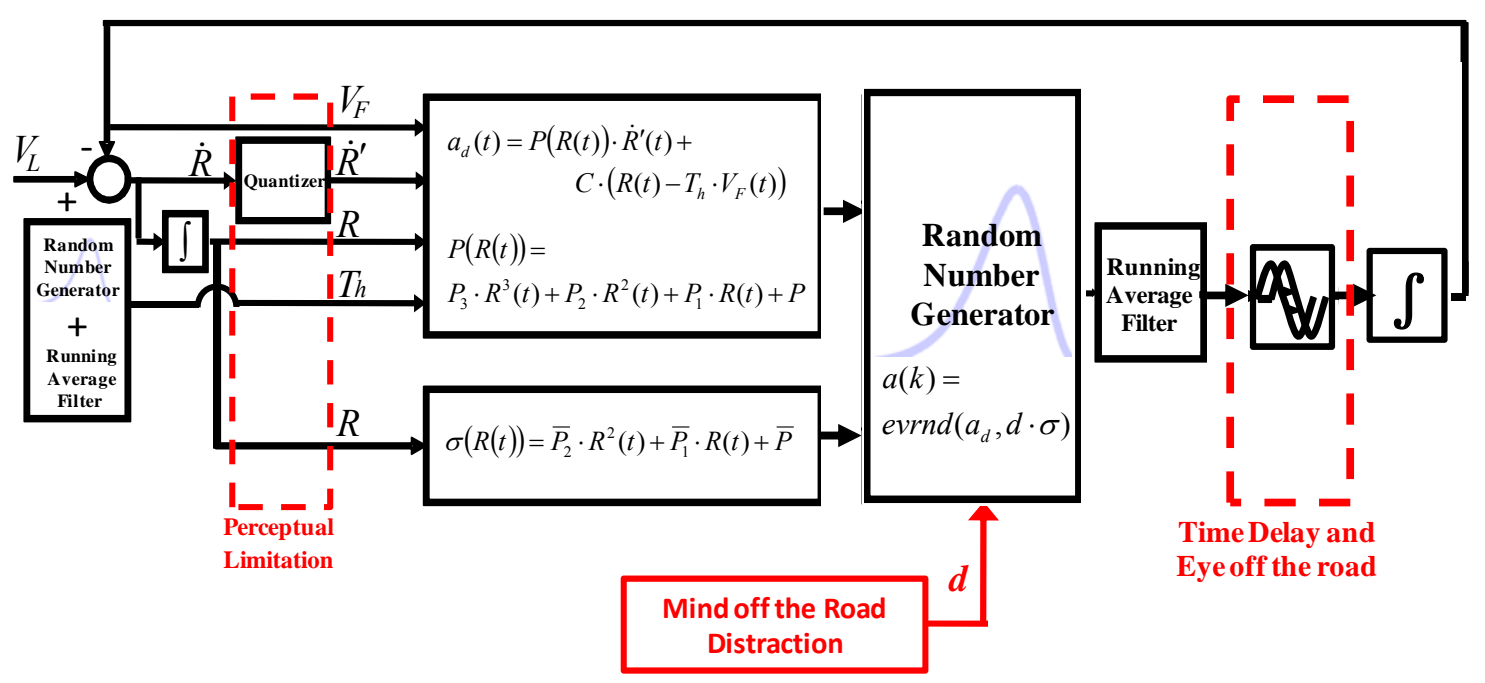

Figure 4.12 Block diagram of the errorable driver model

A time-domain simulation example is shown in Figure 4.13. A minor crash occurs under a lead-vehicle maneuver extracted from the RDCW database. Notice that in this particular case, the simulated driver is much slower than the actual test result, because during the critical moment, the simulated driver happens to have several long delays. If we simulate the same scenarios multiple times, under most cases there will be no crash. The behavior of the simulated driver should be similar to the actual driver statistically.



Figure 4.13 Time domain simulation of errorable driver model 
A thorough validation of the proposed model is hard to do, because of the statistical nature of human drivers and the proposed model. In addition, despite of great promise, advanced tools such as functional MRI is still far away from practical use to assess real human errors. Therefore, crash per Vehicle Miles Traveled is used to define error rate in this paper. To evaluate the crash rate of the errorable driver model, 340 RDCW driving maneuvers of ten drivers were selected and the lead vehicle speed was used as the input to the errorable driver model. Those maneuvers have time-span from fifty to more than three hundred seconds. Each of them was simulated for one thousand times to fully demonstrate the stochastic nature of the model. The simulated crash rate was shown in Table 4.2

Table 4.2 Errorable driver model crash rate

\begin{tabular}{ccc}
\hline Crash Number & $\begin{array}{c}\text { Total Simulated } \\
\text { Miles Travelled }\end{array}$ & Crash/100M VMT \\
\hline 25 & $\mathbf{8 , 8 4 6 , 9 7 6}$ & 282.6
\end{tabular}

This simulation results was compared with actual crash data obtained from NHTSA report [1]. In year 2005, the actual crash rate for rear-end collision is approximately 120/100M VMT. Our simulation crash rate is a little bit higher. One possible reason is that not all minor crashes are reported. Another possible explanation is the lack of a feedback mechanism in our model under near-crash situations. In actual near crash driving, a driver may be warned by passengers, brake light, etc. and engage in an emergent maneuver, with much higher deceleration level, which was not captured in our model. In other words, the errorable driver model developed in this paper represents a conservative approximation of actual drivers, with all statistical characteristics identified from actual driving data. This conservative characteristic, we believe, is actually useful for evaluating AST, such as Collision Warning/Collision Avoidance systems. CW/CA system is designed for situations when the driver does not properly react to a near crash event, which the proposed model seems to emulate. 


\section{CONCLUSION}

An errorable car-following driver model was developed in this paper. A stochastic driver model (SDM) was used as a basis for approximating normal human driving task. In contrast to many driver models in the literature, this SDM contains a stochastic part extracted from real driving data and already captures some of the random nature of human drivers. Subsequently, three error-inducing mechanisms were implemented in the SDM: perceptual limitation, distraction, and time delay. Each of them degrades the driving performance. The perceptual limitation was implemented as a quantization process. The distraction and time delay were modeled as stochastic processes. The parameters of those stochastic processes were estimated from available data in the literature. The resulting crash rate of the errorable driver model is slightly higher than reported NHTSA data (272/100M simulated VMT vs. 120/100M VMT). The possible explanation of this discrepancy are (i) non-reporting of minor crashes in the NHTSA data, and (ii) lack of a feedback mechanisms under near-crash situations in our model. Comparing with conventional car-following driver models that aim to approximate normal human driving, the errorable driver model is more useful for the design and evaluation of active safety vehicle systems.

\section{REFERENCES}

[1] J. Stutts, "Distraction in Everyday Driving", AAA Foundation for Traffic Safety, 2003.

[2] S. G. Klauer, et al, "The Impact of Driver Inattention on Near-Crash/Crash Risk", NHTSA, 2006, DOT HS 810594.

[3] National Highway Traffic Safety Admission (NHTSA), Traffic Safety Facts 2005: A compilation of Motor Vehicle Crash Data from Fatality Analysis Reporting System and the General Estimates System. National Center for Statistics and Analysis, US Department of Transportation, Washington, DC., 2005.

[4] L. A. Pipes, "An Operational Analysis of Traffic Dynamics", in J. Applied Physics, vol. 24, 1953, pp. 271-281.

[5] R. E. Chandler, R. Herman, EW Montroll, "Traffic Dynamics: Studies in Car-following", in Operations research, vol. 6, 1958, pp165-184.

[6] D. C. Gazis, R. Herman, R. W. Rothery, "Nonlinear Follow-The-Leader Models of Traffic Flow", in Operations Research, vol. 9, no. 4, Jul. - Aug., 1961, pp. 545-567.

[7] R Herman, R. B. Potts, "Single Lane Traffic Theory and Experiment.", in Proceeding s of the Symposium on Theory of Traffic Flow, Research Labs, GM, New York: Elsevier, 1959.

[8] J. Treiterer, J. J. A. Myers, "The Hysteresis Phenomenon in Traffic Flow", in Proceedings of the Sixth International Symposium on Transportation Theory, Sydney, 1974, pp 13-38.

[9] H. Ozaki, "Reaction and Anticipation in the Car-following Behavior", in Proceedings of the 13th International Symposium on Traffic and Transportation Theory, pp 349-366.

[10] G. F. Newell, "Nonlinear Effects in the Dynamics of Car-following", in Operations Research, vol. 9, no. 2 1961, pp.209-229. 
[11] W. Helly, "Simulation of Bottlenecks in Single Lane Traffic Flow", in Proceedings of the Symposium on Theory of Traffic Flow, Research Labs, GM, New York: Elsevier, 1959.

[12] J Tyler Jr., "The Characteristic of Model-Following Systems as Synthesized by Optimal Control", in Automatic Control, IEEE Transactions on, vol. 9, issue 4, 1964, pp. 485-498.

[13] G. O. Burnham, J. Seo, G. A. Bekey, "Identification of Human Driver Models in Car-following", in IEEE Trans. on Automatic Control, vol. ACT-9, 1974, pp.485-498.

[14]P. G. Gipps, "Behavioral Car-Following Model for Computer Simulation", in Transport. Res., vol. 15B, no. 2, 1981, pp. 105-111.

[15] M. Bando, et al.," Phenomenological Study of Dynamical Model of Traffic Flow", in J. Phys. I France, vol. 5, pp. 1389-1399, 1995.

[16] R.M. Michael, "Perceptual Factors in Car-Following", in Proceeding of the Second International Symposium on the Theory of Road Traffic Flow, 1963, pp. 44-59.

[17] D. N. Lee, "A Theory of Visual Control of Braking Based on Information about Time-to-Collision", in Perception, vol. 5, no 4, 1976, pp. 437-459.

[18]E. H. Yilmaz, W. H. Warren, "Visual Control of Braking: A Test of the Tau-dot Hypothesis", in Journal of experimental psychology, 1995.

[19]U. Reiter, "Empirical Studies as Basis for Traffic Flow Models", in Proceedings of the Second International Symposium on Highway Capacity, vol. 2 1993, pp. 493-502.

[20] M. Brackstone, B. Sultan, M. McDonald, "Motorway Driver Behavior: Studies on Car-following", in Transportation Research, 2002, pp 329-344.

[21] K. Lee, H. Peng, "Evaluation of Automotive Forward Collision Warning and Collision Avoidance Algorithm", in Vehicle System Dynamics, vol. 43 no. 10, 2005, pp 735-751.

[22]K. Nagel, M. Schreckenberg, "A Cellular Automation Model for Freeway Traffic", in J. Physic I France. vol. 2, 1992, pp. 2221-2229.

[23] S. Krauss, P. Wagner, C. Grawron, "Continuous limit of the Nagel-Schreckenberg model", in Physical Review E, vol. 54 no. 4, Oct. 1996, pp. 3707-3712.

[24]D. Jost, K. Nagel, "Probabilistic Traffic Flow Breakdown In Stochastic Car-following Models", in Transportation Research Record, vol. 1852, 2003, pp. 152-158.

[25]P. Wagner, I. Lubashevsky, "Empirical Basis for Car-Following Theory Development", in Statistical Mechanics, 2006.

[26] S. J . Brunson, E. M . Kyle, N. C. Phamdo, G. R. Preziotti, "NHTSA Rear-End Collision Alert Algorithm Final Report", NHTSA, 2002, DOT HS 809526.

[27]D. LeBlanc, "Road Departure Crash Warning System Field Operational Test: Methodology and Results", Transportation Research Institute (UMTRI), University of Michigan, Ann Arbor, MI, DTFH61-01-X-0053, 2006, vol. 1.

[28] A. M. Mood, F. A. Graybill, D. C. Boes, "Introduction to the Theory of Statistics", 3rd edition, McGraw-Hill, 1974, pp. 540-541.

[29] H. K. Khalil, "Nonlinear Systems", third Ed., Prentice Hall. 2000, pp.551-571.

[30] M. S. Chang, C. J. Messer, A. J. Santiago, "Timing Traffic Signal Change Intervals Based on Driver Behavior", in Transportation Research Record, vol. 1027, 1985, pp. 20-30.

[31]R. Dewar, P. Olson, "Human Factors in Traffic Safety", $2^{\text {nd }}$ ed., Lawyers \& Judges Publishing Company, Inc.

[32] R. Henderson, A. Burg, "Vision and Audition in Driving, Final Report", NHTSA, 1974, DOT HS 801265.

[33] J. J. Gibson, “The Ecological Approach to Visual Perception”, Boston: Houghton Mifflin, 1979.

[34]D. R. Proffitt, "Distance Perception", in Current Directions in Psychological Science, vol. 15, no. 3, 2006, pp131-135.

[35] K. Nakayama, C.W. Tyler. "Psychophysical Isolation of Movement Sensitivity by Removal of Familiar Position Cues", in Vision Research, vol.21, 1981, pp. 427-433.

[36] S. P. McKee, “A Local Mechanism for Differential Velocity Detection”, in Vision Research, vol. 21, 1980, pp 491-500.

[37]R. H. Brown," Weber Ratio for Visual Discrimination of Velocity", in Science, vol. 131. no. 3416, 1960, pp. 1809-1810.

[38] J. M. Harris, S. N. J. Watamaniuk, "Speed Discrimination of Motion-in-Depth Using Binocular Cues", in Vision Research, vol. 35, no. 7,1995, pp. 885-896. 
[39] S. N. J. Watamaniuk, "Perceptual and Oculomotor Evidence of Limitations on Processing Acceleration Motion", in Journal of vision [1534-7362] vol. 3 issue. 11, 2003 pp. 698 -709.

[40] T. Haarmeier, P. Their, "Detection of Speed Changes during Pursuit Eye Movement", in Exp. Brain Res, 2006, 170: 345-357.

[41] J. Stutts, "The Role of Driver Distraction in Traffic Crashes", AAA Foundation for Traffic Safety, 2001.

[42] T. B. Sheridan, "Driver Distraction From a Control Theory Perspective", in Human Factor, vol. 46, no. 4, 2004, pp. 587-599.

[43]E. R. Boer, "Behavioral Entropy as a Measure of Driving Performance", in Proceeding to Driver Assessment, 2001, pp225-229.

[44] S. Ross, "Stochastic Processes", $2^{\text {nd }}$ ed. Wiley.

[45]A. Pick, D. Cole, "Neuromuscular Dynamics and the Vehicle Steering Task", in Vehicle System Dynamics. vol. 41, 2004, pp. 182.

[46] O. Tsimhoni, H. Fuller, M. Reed, "Modeling In-Vehicle Display Position and Driver Performance”, in Annual Meeting Industry Affiliation for Human Factors in Transportation Safety.

[47] M. Lesch, L. Simmons, P.A. Hancock, "The Distraction Effects of Phone Use during a Crucial Driving Maneuver", in Accident Analysis and Prevention, 2003.

[48] H. Alm, L. Nilsson, "The Effects of a Mobile Telephone Task on Driver Behavior in a Car-Following Situation", in Accident Analysis and Prevention, 1995.

[49] D. Lamble, et al, "Cognitive Load and Detection Thresholds in Car-Following Situations: Safety Implication for Using Mobile Telephone While Driving”, in Accident Analysis and Prevention, 1999. 